Review

\title{
Biological Homochirality on the Earth, or in the Universe? A Selective Review
}

\author{
Vadim A. Davankov \\ Institute of Organo-Element Compounds, Russian Academy of Sciences, 119991 Moscow, Russia; \\ davank@ineos.ac.ru
}

Received: 21 November 2018; Accepted: 10 December 2018; Published: 13 December 2018

\begin{abstract}
The discovery of meteoritic alpha-amino acids with significant enantiomeric excesses of the L-form has suggested that some cosmic factors could serve as the initial source for chiral imbalance of organic compounds delivered to the early Earth. The paper reviews major hypothesis considering the influence of chiral irradiation and chiral combinations of physical fields on the possible ways asymmetric synthesis and transformations of organics could take place within the solar system. They could result in a small enantiomeric imbalance of some groups of compounds. More attention is paid to the hypothesis on parity violation of weak interaction that was supposed to cause homochirality of all primary particles and a more significant homochirality of compounds directly synthesized from the latter in a plasma reactor. The first experiment with material synthesized in a plasma torch resulting from a super-high-velocity impact showed formation of alanine with the excess of L-form between 7 and $25 \%$. The supposed conclusion is that L-amino acids could serve as a starting homochiral biomolecular pool for life to emerge all over the Universe.
\end{abstract}

Keywords: origin of life; biological homochirality; deracemization; super-high-velocity impact; plasma reactor; absolute asymmetric synthesis; amino acids

\section{Introduction}

The recently revised estimates of the age of our Earth amount to 4.54 billion years. Some 4.3 billion years old rocks already show traces of water withering, implying the existence of oceans and land. Surprisingly, isotopic analysis of some zircon crystals, 4.1 billion years old, reveals carbon-rich inclusions which allow suspecting the existence of primitive organic life at that time. In any case, it did not take long, just a few hundred million years, for life to emerge on the young Earth. This could only be possible if abundant organic matter with a predominant homochirality has been accumulated in its aqueous basins. Indeed, there is no doubt that numerous organic compounds have been transported to the planet's surface or were formed on it during the times of intense bombardment of planets with meteorites. Even now, the Mars rover "Curiosity" detects organic molecules in the Gale Crater, a depression that was most likely a freshwater lake about 3.5 billion years ago. Among such compounds are thiophenes and dimethyl sulfides, but also aromatic compounds, such as toluene, chlorobenzene, and naphthalene, as well as chain hydrocarbons. The soil samples examined by Curiosity come from the top $5 \mathrm{~cm}$ of the Martian surface and thus they survived in a chemically very aggressive and hostile environment exposed to cosmic rays and the ionizing and oxidizing conditions [1]. Where the molecules come from; whether they have been delivered from elsewhere or directly formed on the planet's surface is unclear.

As early as 1988, Gol'danskii and Kuz'min [2] convincingly proved that abundance of organic matter is the precondition that is required but not sufficient for life to emerge. Biopolymers present numerous high-molecular-weight compounds whose unique three-dimensional structures are absolutely essential for their productive functioning during their interaction with other components of 
a living cell. This uniqueness of each biopolymer structure is guaranteed not only by the unambiguous sequence of their building blocks-alpha amino acids, sugars, and nucleotides-but also by the homochirality of basic classes of compounds with asymmetric molecules, such as amino acids and sugars. The problem of the emergence of life thus cannot be solved without identifying mechanisms providing homochirality of the above series of compounds, since without preexisting homochirality, the self-replication characteristic of living matter could not occur in principle. No racemic primordial soup of organic compounds would ever give rise to any self-replicating system. Shandrasekhar [3] reasonably notes: "It is indeed sobering - if not depressing - to consider the fact that, to the extent that a firm answer to the question on the origin of molecular chirality does not emerge, the origin of life will remain a mystery."

Numerous literature exists dealing with the emergence or enhancement of chirality in a racemic system by a so-called chance mechanism: spontaneous resolution on sublimation or crystallization with or without Viedma ripening [4], spontaneous symmetry breaking via stereospecific autocatalysis, asymmetric adsorption, or asymmetric synthesis on chiral crystals. Some of the above processes can give, within a rather small location, individual products having very high enantiomeric purity of an undetermined sign, with a similar probability for creating the opposite stereochemical result in another small location. None of the chance processes could thus provide a global predominance of L-enantiomers for the whole series of amino acids and D-isomers for sugars. Besides, it is quite impossible to imagine the above processes to proceed efficiently in the complex matrix of the primordial soup of organic compounds. Therefore, if the chance scenario for the origin of abiotic chiral purity is not viable, then all arguments strongly favor theories of induced extraterrestrial homochirality of initial organic matter.

Searching for chiral organic matter in our solar system is a major challenge for the space research community and has attained increasing attention from many research groups. Review [5] analyzed functional possibilities generated by numerous sophisticated instrumentation and technologies available or being developed especially for space survey missions. One advanced space mission, namely, the Rosetta spacecraft, after a 10-years-long journey, detached in November 2014 the Philae lander on the surface of comet 67P/Churyumov-Gerasimenko (67P/C-G). The instrument was fully prepared to conduct for the first time an extraterrestrial stereochemical investigation of a soil extract by employing a multicolumn gas chromatograph and a time-of-flight mass spectrometer [6]. After methylation with $\mathrm{N}, \mathrm{N}$-dimethylformamide dimethyl acetal, a series of chiral molecules, such as amino acids, hydrocarbons, amines, alcohols, diols, and carboxylic acids, could be analyzed. Unfortunately, the experiment failed because of insufficient energy supply, so that no enantiomeric on-site analysis has been performed thus far on any organic extraterrestrial material. On the contrary, numerous laboratory works have been conducted in many countries simulating possible sources of extraterrestrial organic matter, including chiral compounds.

\section{Origins of Extraterrestrial Non-Racemic Organic Matter}

Since Miller's seminal works from 1953 and later, it has become clear that specific biological building blocks could well be formed in the atmosphere of any planet from a primitive reducing gas mixture of water, methane, and ammonia, but also from the later suggested gas composition consisting mostly of carbon dioxide, nitrogen, and water. For instance, both UV and charged particle irradiation of simple gas mixtures of $\mathrm{H}_{2} \mathrm{O} / \mathrm{CH}_{3} \mathrm{OH} / \mathrm{NH}_{3} / \mathrm{CH}_{3} \mathrm{CN}$ were shown to produce up to 26 racemic amino acids, diamino acids, and $\mathrm{N}$-(2-aminoethyl)glycine [7], but also purine and pyrimidine compounds, urea, and polyols, along with other prebiotic molecular structures [8].

Simultaneous formation of many more important organic compounds, namely, that of nucleobases (cytosine and uracil), various proteinogenic amino acids (glycine, alanine, serine, aspartic acid, glutamic acid, valine, leucine, isoleucine, and proline), non-proteinogenic amino acids, and aliphatic amines, was reported in experiments simulating reactions induced by extraterrestrial objects impacting on the 
early oceans [9]. The latter are expected to have had sufficient amounts of dissolved bicarbonates and inorganic nitrogen as the sours of required elements.

Another medium suitable for emergence of organic molecules is cosmic ice, even at extremely low temperatures. The latter are beneficial to the survival of molecules that were formed in space by one mechanism or another. In general, individual molecules and heavier atoms are assumed to tend to gather or adhere to already existing cold solid dust material. Since water is one of most abundant compounds in the interstellar space, formation of ice bodies with the incorporation of larger molecules and dust particles is a very common process. Indeed, astrophysical ices contain simple molecules $\left(\mathrm{CO}_{2}\right.$, $\mathrm{H}_{2} \mathrm{O}, \mathrm{CH}_{4}, \mathrm{HCN}, \mathrm{NH}_{3}, \mathrm{CO}$, and others). Once formed, the ices are exposed to complex radiation fields, e.g., UV, $\gamma$ - and X-rays, stellar/solar wind particles, cosmic rays, and collisions with other cosmic bodies. It is only natural to assume that cosmic ices not only entrap various larger organic molecules but also present a suitable matrix for the formation of new ones, e.g., via radical mechanisms. Indeed, glycine (Gly), the simplest amino-acid building-block of proteins, has been identified on icy dust grains in the interstellar medium, icy comets, and ice-covered meteorites, as in the comet 81P/Wild-2 samples collected and returned to Earth by NASA's Stardust spacecraft.

Martins et al. [10] presented results of laboratory experiments in which ice mixtures analogous to those found in a comet were subjected to a shock with a steel projectile fired at a hypervelocity using a light gas gun. The hypervelocity impact shock of a typical comet ice mixture was found to produce several amino acids after hydrolysis. These included equal amounts of D- and L-alanine, and the non-protein amino acids $\alpha$-aminoisobutyric acid and isovaline as well as their precursors. Simulation of astrophysical conditions by exposure of multilayer $\mathrm{CO}_{2}: \mathrm{CH}_{4}: \mathrm{NH}_{3}$ ice films to $0-70 \mathrm{eV}$ electrons also revealed formation of multiple products, among them glycine [11]. Other amino acids could well form too, though in smaller quantities.

The discovery of meteoritic $\alpha$-amino acids with significant enantiomeric excesses of the L-form has suggested that some cosmic factors could serve as the initial source for chiral imbalance of organic compounds. In this case, the non-racemic extraterrestrial organic materials delivered to Earth by carbonaceous meteorites (chondrites) may have contributed to prebiotic chemistry and finally directed to homochirality of amino acids and carbohydrates on Earth. Chondrites present remnants of asteroids that survived extreme heating in the Earth's atmosphere and impact with the solid surface. Chondrites contain numerous extractable organic compounds and, in even larger amounts, insoluble crosslinked polymeric kerogen-like organic material. As summarized in the review paper by the group of $d^{\prime}$ Hendecourt and Meierhenrich [6], extensive studies of the Murchison and Murray meteorites "revealed the presence of a series of alkyl-substituted bicyclic and tricyclic aromatic compounds, aliphatic compounds ranging from $\mathrm{C} 1$ to $\mathrm{C} 7$, including both saturated and unsaturated hydrocarbons, more than 80 amino acids, including diamino acids, $\mathrm{N}$-alkylated amino acids and iminodiacids, small amounts of aldehydes and ketones up to C5, a wide spectrum of carboxylic acids and hydroxycarboxylic acids, several nucleobases, and sugar acids." Thank to available sensitive chromatographic techniques for enantiomeric analysis, from the above diverse array of meteoritic organic molecules, four groups of compounds having asymmetric carbon atoms-namely, amino acids, hydroxycarboxylic acids, monocarboxylic acids, and amines-have been examined for their stereochemical composition. All amino acids were found to display the predominance of one type of configuration with a wide range of $e e$ values. $\alpha$-Substituted amino acids, which are known to be especially resistant to racemization, exhibited extremely high enantiomeric excess: up to $60 \%$ for D-alloisoleucine [12] and 18\% for L-isovaline [13]. Surprisingly, meteoritic aliphatic amines and carboxylic acids that might share a common chemical origin with amino acids were found to be racemic with the exception of $\alpha$-hydroxypropionic (lactic) acid [14]. According to Soai et al. [15], the insoluble and resistant to hydrolysis meteoritic material also incorporates chiral fragments.

Several mechanisms for the emergence of extraterrestrial chirality have been considered and subjected to experimental testing. Rather popular is the idea of deracemization of pre-formed racemic amino acids, i.e., distortion of the initial 1:1 proportion of their enantiomers. A number 
of deracemization scenario are discussed involving chiral irradiation, chiral combinations of physical fields, and beams of chiral particles, all of which are known to be emitted by nascent neutron stars, short supernova bursts, or growing black holes. Indeed, a supermassive black hole, billions of times heavier than the Sun, spinning at the galactic center shoots out two very intensive narrow jets of matter and chiral radiation in opposite directions [16].

As was shown, circularly polarized irradiation may cause slightly enantioselective destruction (deracemization) of racemic compounds. Numerous extensive papers and reviews analyze astronomical sources of circularly polarized light $[17,18]$ and results of model experiments on deracemization of amino acids $[19,20]$ in terms of possible origins of biological homochirality on the Earth $[6,21]$.

Besides deracemization of pre-formed amino acids, direct asymmetric synthetic processes can take place on space objects exposed to circularly polarized irradiation. Thus, in circumstantial laboratory experiments on model achiral extraterrestrial ice [22,23], the asymmetrical synthesis of amino acids was found to take place under irradiation with visible and UV circularly polarized light (CPL) at two different photon energies (6.6 and $10.2 \mathrm{eV})$. Sixteen distinct amino acids were identified and the enantiomeric composition of five of them ( $\alpha$-alanine, 2,3-diaminopropionic acid, 2-aminobutyric acid, valine, and norvaline) was precisely measured using the enantioselective two dimensional gas chromatography-time of flight mass spectrometry technique technique. The results obtained on such irradiated ices showed certain symmetry breaking of amino acids with the ee values rising with time from $e e \mathrm{~L}=-0.20 \% \pm 0.14 \%$ to $e e \mathrm{~L}=-2.54 \% \pm 0.28 \%$. The sign of the induced $e e$ (that depends on the helicity of the CPL) was the same for all five considered amino acids and did not depend on the evolutionary stage of the samples. Surprisingly, the chirality sign was also found to depend on the energy of the CPL.

Similar formation of slightly enantiomerically enriched amino acids was earlier considered by irradiation of their initially racemic pools with intensive antineutrino streams [24]. More recent works $[25,26]$ suggested that the deracemization of the amino acids could be established due to the magnetic field of a nascent neutron star from a core-collapsed supernova via amino acid processing by the neutrinos that would be emitted. A theoretically derived supernova neutrino amino acid processing model, or SNAAP model, not only appears to produce a small chiral imbalance, but, importantly, always produces the same sign of the chirality for different amino acids [27].

Chirality also emerges when subjecting thin films of amino acids to polarized light from synchrotron radiation and a free electron laser. This process is thought to mimic the evolution of organics adsorbed on the surface of interstellar dust particles [28].

Finally, spin-polarized electrons emitted by the $\beta$-decay of radioactive nucleons were also shown to cause ionization of two enantiomeric molecules of amino acids at slightly different rate thus resulting in small enantiomeric enhancement of the last surviving portions of the initial racemic material. This effect known as "electronic circular dichroism" was applied to induce small optical activity in leucine [29], tryptophan [30], and alanine [31]. However, the kinetic energy of the investigated spin-polarized electrons was by orders of magnitude too high for efficient enantiomer-discriminating absorption, which results in almost total destruction of the initial portion of amino acids.

All the above considered determinate mechanisms of creating homochiral groups of organic compounds (which include symmetry breaking induced by combinations of circularly polarized irradiation, and magnetic or even gravitational [32] fields) expand the search area for origins of chirality and life from some locations on the Earth to the interplanetary matter existing in space regions as big as our Solar system.

Indeed, a more or less expressed enantioselective interaction between organic matter and various physical fields and elementary particle beams seems to be a much more common phenomenon than has been earlier expected. Just to mention that electron transmission through chiral molecules was found to depend on the electron spin, and thin films of chiral compounds, in particular nucleic acids and peptides, have been shown to act as spin filters, as summarized in the recent review [33]. 
Still, all the above hypotheses of extraterrestrial origins of biological homochirality on Earth suffer from several important weak points.

First of all, for life to start on Earth, it is critical that the building blocks of amino acids, sugars, and nucleosides be created in space in homochiral form, namely, with a predominant L-configuration for all amino acids and D-configuration for all sugars. This unique direction of enantioselectivity is not confirmed for the action of all types of neutrino, antineutrino, polarized electrons, or streams of other cosmic particles. As for the rays of electromagnetic waves, in different area of space, they may have different signs of circular polarization. Up to now, a relatively large (from $+17 \%$ to $-5 \%$ ) circular polarization of irradiation (namely in the IR range of radiation) was reported from the Orion nebula OMC-1 star-forming region extending about 400 times the size of the solar system [34].

Notably, such CPL sources will not usually generate a net circular polarization because they will have regions of positive and negative sign that cancel when averaged over any galactic-sized area like the Milky Way. The discussed irradiation scenario for the origin of homochirality requires that the Sun system be formed during only one single chiral cosmic event dominating over a very large region of a few parsecs in size. The hypothesis involves development of the planetary system in a high-mass star-forming region where matter was subjected to the influence of an external source of CPL or chiral combination of physical fields of a given helicity and a favorable dominant energy during the protoplanetar phase of evolution, thus inducing one definite stereo-specific photochemistry in the system. That the solar system originated in such a massive star formation region is supported by isotopic studies of meteorites' compositions, such as those including ${ }^{60} \mathrm{Fe}$, suggesting that a supernova explosion occurred near the Sun [35].

Another general problem is that the configuration of amino acids induced by circularly polarized irradiation depends on the energy of its quanta, i.e. on its wavelength [23]. Photolysis of amino acids requires UV radiation, rather than the infrared radiation observed in the Orion massive star forming area. Of course, UV radiation cannot be directly observed as it is unable to penetrate the dust that lies along the line-of-sight between the Earth and regions of highly polarized radiation activity. The general stereochemical outcome of irradiation with CPL of the whole pallet of amino acids was critically analyzed by Cerf and Jorissen [36]. When taking into account that asymmetric photolysis implies the preferential destruction of the enantiomer having the higher absorption coefficient, the highest stereodifferentiation efficiency of irradiation is directly related to the circular dichroism (CD) band of the compound. For amino acids, the carboxyl group bound to the alpha-carbon has a strong $\mathrm{CD}$ band centered at about $210 \mathrm{~nm}$. The sign of this CD band is the same for all aliphatic L-amino acids. It is not the case for tryptophan [37], whose indole chromophore exhibits a strong CD band centered at about $195 \mathrm{~nm}$, with the opposite sign to the carboxyl $210 \mathrm{~nm}$ band. Proline also has a strong CD band of opposite sign around $193 \mathrm{~nm}$ in a neutral solution. Therefore, these two amino acids would violate the homochirality of the pool as a whole if the asymmetry would arise from the enantioselective photolysis of all the racemates with CPL with the wave length around $200 \mathrm{~nm}$. At the same time, there seems to be no spectral window where all the biogenic amino acids have a strong CD band of one and the same sign. Besides, the intensities and signs of $\mathrm{CD}$ bands depend on the properties of the medium, so that extrapolation of laboratory data obtained in liquid solutions to infer the CD properties of amino acids in space (where they are likely to be found in solid or gas phase) is not straightforward.

It follows from the above considerations that the energy of the circularly polarized UV irradiation or particle beams must fit into a very narrow range in order to cause efficient ionization and stereochemical transformations of organic molecules resulting in the deracemization of preformed compounds or asymmetric synthesis of new ones. Less energetic impacts are inefficient while impacts of higher energy will totally destroy larger organic molecules. In fact, no circularly polarized cosmic radiation in the UV region that is required to trigger desired chemical transformations has been detected thus far. As a rule, cosmic rays of accelerated particles and radiation are destructive to unprotected organics rather than stimulating efficient synthetic processes. Maybe, cosmic ice bodies present exclusion due to the existence of some layers with reduced energies of radiation and emergence 
of secondary electrons. On the other hand, the extent of circular polarization of their impacts still remains to be examined. Also, one should bear in mind that we deal with dynamic processes where surface layers of ice bodies partially evaporate while they experience bombardment with cosmic rays.

When taking into account the predominant destructive role of all cosmic rays, it is difficult to expect any significant accumulation of chiral biomolecules on unprotected supports, the more that a measurable degree of enantioenrichment is only attained for the last small portion of the initial material. Even then, $e e$ levels attained for these last portions of amino acids could not exceed few per cent. These values are by orders of magnitude smaller than what is observed in chondritic meteorites. All these considerations lead to a suggestion that the major part of homochirally enriched organic molecules were synthesized directly on the atmosphere-protected planets, rather than delivered to them by radiation-exposed cosmic dust particles and ice bodies.

\section{Parity Violation in the Weak Interaction and Homochirality of Matter}

The above-discussed processes with determinate origins of chirality could possibly generate very small chirality within regions as large as the solar system, but only as a result of one single cosmic event, such as a supernova burst, since any second similar burst event could generate the opposite sign of chirality of all enantioselective processes. The only universal and constant source of invariant chirality thus remains the phenomenon called violation of parity in the weak interaction.

Parity conservation implying that nature is symmetrical under reflections in a mirror enjoyed the status of a fundamental law of physics along with those of conservation of energy, momentum, and electric charge. Yet, although there were many experiments that established parity conservation in strong interactions, the weak force, which was first postulated to explain disintegration of elementary particles, was shown to violate the parity conservation law. It was demonstrated on radioactive ${ }^{60} \mathrm{Co}$ that the $\beta$-decay of nuclei oriented with a strong magnetic field emits exclusively left-polarized electrons, which unequivocally violates parity expectations $[38,39]$. This discovery revolutionized our understanding of nature's fundamental laws. As one of its consequences, we must expect a small energy difference between any two molecular structures that we used to call enantiomers. The energy gap between them was calculated to have an order of $10^{-12}-10^{-15} \mathrm{Jmol}^{-1}$ [40] with a magnitude and sign that depend on the particular molecule and, unfortunately, also on its conformation. This energy difference is too small to be measured and also too small to cause any measurable enantiomeric enrichment under conditions of thermodynamic equilibration of a racemate.

Nevertheless, the role of parity violation in the creation of initial partial homochirality of organic matter under kinetic conditions, far from a thermodynamic equilibrium, must be considered more precisely. According to Davankov [41], parity violation in $\beta$-decay processes may be interpreted in terms of the chirality of elementary particles and is closely related to the phenomenon of the experimentally observed chirality of atoms [42], assuming the difference in terminology used in chemistry and physics is neglected. Since the discovery of parity violation, physicists now and then, analyzed the idea of "how chiral symmetry, its pattern of breaking and restoration under extreme conditions manifest themselves in the nucleon, nuclei, nuclear matter and hadronic matter" [43]. Even now, asymmetry at the level of atoms and elementary particles remains both a wonder and a challenge. In principle, it may give a valuable hint toward something new in the standard model and reconsiders the role of neutrinos, which are elusive particles that are difficult to detect. Moreover, current measurements of violation with respect to simultaneous space reflection and charge conjugation (charge-parity $(\mathrm{CP})$ violation) in the decay of B mesons, which is the phenomenon of asymmetry itself, appear to account for the excess of matter over antimatter [44,45].

Recently, the European Space Agency's Planck telescope finished examining the cosmic microwave background, the faint afterglow of the Big Bang, so that final maps of the early Universe could be adjusted and released. These data helped researchers to pin down the age of the Universe (about 13.8 billion years), and its composition ( $95 \%$ dark matter and dark energy). The observable $5 \%$ of mass, which is the Universe, is made of matter, not antimatter. This means, that soon after Big Bang, particles 
would decay somewhat slower than their counterparts, antiparticles, and "CP violation" in particle decays could be the reason for that. It is assumed that stability of particles and their clusters is related to their weak charges. Thus, the proton's weak charge defines the strength of certain interactions between protons and other particles. More recently, scattering of left-polarized and right-polarized electrons on protons was precisely measured. The difference between the two measurement results characterizes the weak current of protons, its asymmetry, and was found to amount to $-226.5 \pm 9.3$ parts per billion, where the minus sign indicates that left-handed electrons are more likely to be scattered than their right-handed counterparts. The corresponding proton's weak charge is $0.0719 \pm 0.0045$, while the proton's electric charge is +1 . To put the magnitude of the above asymmetry in perspective: if parity symmetry were violated for the height of mountains, Mount Everest and its mirror-image twin would differ in height by a mere 2 millimeters [46]. Nonetheless, the important message in terms of chemistry is: protons are chiral.

Earlier, Davankov [47] presented a more common argument for the chirality of another elementary particle, the electron. It is known that any electric current, that is propagation of electrons along a wire (or just in vacuum), is accompanied with circular magnetic field of one definite direction. This makes the whole system, moving electrons/circular magnetic field, invariantly chiral. The intensity of the field only depends on the number of electrons that pass through the wire cross section during a unit time, but not on the creep velocity of electrons along the wire that would depend on voltage applied. One could argue that the voltage drop may cause partial spin-polarization of electrons. However, the velocity of random movements of electrons within a metal at room temperature exceeds the creep velocity by ten orders of magnitude, such that any spin polarization at that large ratio of rates appears highly improbable. The only known source of the invariant chirality of the system is the inherent chirality of electrons. It is only natural that any moving charged particle, both negative and positive, combined with the corresponding magnetic field, would compose a system with one definite sign of chirality. This leads to a conclusion that every charged elementary particle is inherently chiral. Importantly, any antiparticle having an opposite charge, like in the pair electron/positron, will produce an opposite chirality. For this reason, Davankov's suggestion [47] is that charged particles and their antiparticles have the opposite inherent chirality sign. This suggestion of some kind of enantiomeric relationship was further extended to all pairs of neutral particles and antiparticles.

What is even more important, Davankov formulated a general hypothesis [41,47] that all elementary particles in our universe compose a homochiral pool of which all atoms and molecules are built, while all elementary antiparticles would compose a homochiral pool that formed antimatter. The reality is that the collision of all particles with corresponding antiparticles results in their annihilation with an emission of electromagnetic impulses, i.e. photons of varying energy up to gamma quanta and neutrino, all of which are chiral as well. (According to Davankov [47] each individual photon and each energy quantum are chiral, but, contrary to elementary particles, they can exist in both left-rotating and right-rotating forms). The early universe is theorized to have spawned the exact same amounts of normal matter and antimatter. Since we observe only matter and no consolidated antimatter, two kinds of particles must have played by slightly different rules, letting a fraction of normal particles outlast their bizarre enantiomeric twins. Therefore, scientists are looking for theoretical particles called the axions that could possibly explain the curious asymmetry of the Universe and the massive cosmic mystery of dark matter. Theory says that axions will be absurdly light and hardly interact with normal matter. Until above fundamental theoretical problems receive their explanation, our notion of homochirality of all elementary particles has its right to exist and should be seriously taken into account.

Basically, this hypotheses states that inherent homochirality of normal matter was established in the whole universe soon after the Big Bang, about 13.8 billion years ago, and invariantly subsists for the whole period of its existence. It is only natural to suggest that homochirality of elementary particles exerts certain invariable influence on the overall chirality of atoms. The influence of parity violation could be much less expressed in organic molecules. Chirality of molecular structures belongs 
to a completely different hierarchy of chirality phenomena along with morphological chirality of living organisms or that of crystals. Besides, organic molecules were constantly formed and destroyed during prebiological chemical evolution of matter according to laws of organic chemistry, rather than that of physics.

Still, one can speculate that the above postulated homochirality of elementary particles and atoms could reveal itself to the most significant extent in the processes of the direct synthesis of asymmetric organic molecules from ionized atoms and electrons, rather than from transformations of simple organic precursors. Such direct synthetic processes may proceed during dissipation and the fast cooling of plasma that contain electrons and all required atoms, such as $\mathrm{C}, \mathrm{N}, \mathrm{H}$, and $\mathrm{O}$, in their ionized form. In any event, all numerous simple organic molecules in the universe [5] have emerged in exactly these kinds of processes.

According to Managadze [48], the major part of the more complex organic matter in the universe was also synthesized by collisions between solid cosmic bodies at relative velocities above $15-20 \mathrm{~km} \mathrm{~s}^{-1}$, so-called super-high-velocity impacts. The latter bring about a super-quick heating of the interacting regions of both the target and the projectile up to temperatures of $10^{6}{ }^{\circ} \mathrm{C}$, which results in a total atomization and $100 \%$ ionization of the affected matter. The initially solid matter converts into high-density and high-temperature plasma. The latter dissipates in the form of a so-called plasma torch or burst and rapidly cools down. During the process of the adiabatic expansion of plasma, recombination of ionized atoms and electrons proceeds with the eventual combination of the organic-bearing elements $\mathrm{C}, \mathrm{H}, \mathrm{O}, \mathrm{N}$, and other abandoned elements of matter having a particularly high affinity for each other. They finally combine into both simple and more complex organic compounds including those having centers of asymmetry. A significant part of organic compounds could thus be formed directly on the early Earth's surface (and everywhere else), as well as delivered by meteorites, which would have been especially intense during the first 500 million years after formation of solar planetary system [48].

Davankov's idea is that the general inherent homochirality of elementary particles should manifest itself to the most obvious extent in a kind of direct absolute asymmetric synthesis of first organic molecules and result in the invariant predominant homochirality in all classes of similarly constructed molecules. Notably, the nonequilibrium synthetic processes considered proceed under unsteady flows of colliding primary components in open systems that are far from equilibrium. If this hypothesis is true, amino acids enriched in L-enantiomers and sugars enriched in D-enantiomers have existed in the universe since the times where sufficient solid material has been accumulated in space to form planetary systems around numerous suns. The extent of enantioenrichment of organic molecules on each particular spot should depend on the conditions of their formation in super-high-velocity impacts and their subsequent transformations.

It further follows from the assumption of general homochirality of matter and that of initially formed biomolecules that preconditions for life to emerge do exist everywhere in the universe and that everywhere proteins, sugars, and nucleic acids must have the same unequivocal homochirality sign as that in living organisms on the Earth. At that, the pallet of life-forming building blocks and hereditary codes selected for living matter could well be different on different planets. In other words, the homochirality sign of living matter is predetermined by the inherent universal parity violation on the level of elementary particles, whereas amino acid and sugar compositions of biopolymers could be a matter of chance.

Among the above several fundamental predictions that follow from the hypothesis on homochirality of elementary particles, one could be put to the test by using already existing experimental techniques. It reads that any direct self assembling of $\alpha$-amino acid molecules from ionized atoms and electrons must result in a noticeable homochiral enantiomeric enrichment of the products with predominance of molecules having L-configuration. Arguments for this supposition have been outlined in details by Davankov [47,49] and Managadze [50] in their conceptual publications. 
The main idea of the designed experiment consists in creating of a plasma torch reactor by a super-high-velocity impact of a projectile against a suitable target. Both of them must contain elements required for the formation of amino acids [51]. The dissipating and cooling-down plasma was expected to act as a reactor for the self-assembling of ions, radicals, and atoms, all of them being inherently homochiral, into enantiomerically enriched organic molecules. The plasma torch itself, as a flow of chiral electrons, followed by a flow of heavier, chiral, positively charged particles, presents a reactor with very strong chiral combinations of magnetic and electric fields, as well as intensive circularly polarized light beams. Since the chirality sign of all the components and fields within the plasma torch is invariantly determined by the homochirality of all primary particles, amino acids, if formed at all, are expected to be enriched in L-isomers, especially if the chirality of all building elements act in concert with the induction caused by the chirality of the fields.

As a substantial corroboration of the above-outlined expectation, the striking fact was considered that all amino acids, including nonnatural $\alpha$-methylated amino acids, in meteorites (some of them being much older than Earth) are found to exhibit a predominance of L-isomers with ee as high as $15-18 \%$ (for the racemization-resistant isovaline).

A large international team of scientists [51] started experiments with high purity ${ }^{13} \mathrm{C}$ carbon black that was converted, via the action of high temperatures and high pressure, into diamond particles of about $1.5-2.5 \mathrm{~mm}$ in size. The latter served as projectiles for a super-high-velocity impact experiment and were expected to completely vaporize and label the plasma synthesis products with high concentration of the rare ${ }^{13} \mathrm{C}$ carbon isotope. A tablet of ammonium nitrate served as the $\mathrm{O}, \mathrm{H}, \mathrm{N}$-providing impact target. Since its mechanical properties are week, a strong plate of pure ${ }^{12} \mathrm{C}$ graphite was positioned behind the target. To easily collect the products of the impact, the target was placed into a steel container lined with a titanium foil. The hypervelocity impact experiments were performed using a ballistic launcher equipped with a light-gas gun [52]. The gun accelerated a set of 20-50 diamond projectiles, about $0.4 \mathrm{~g}$ in total weight, to a velocity as high as $7 \mathrm{~km} / \mathrm{s}$. The impact products presented dark gray fine powder, composed of both plasma torch synthesis products and debris of the graphite plate. The latter was $5.6 \mathrm{~g}$ in weight, so that the ${ }^{12} \mathrm{C} /{ }^{13} \mathrm{C}$ ratio in any organic matter synthesized would be 14 if complete mixing of diamond and graphite carbon atoms occurred. One portion of organic material $(2 \mathrm{mg}$ ) was extracted from the impact sample with $2 \mathrm{M}$ hydrochloric acid at $150{ }^{\circ} \mathrm{C}$ for $24 \mathrm{~h}$ and subjected to careful mass-spectrometric investigation. Three separate analytical instruments with different strengths, limitations, and detection limits were used: a matrix assisted laser desorption ionization (MALDI) TOF instrument, a laser desorption TOF instrument, and a GC-MS instrument. In the mass-spectra obtained, the three simplest amino acids and their typical fragmentation ions could be safely observed: glycine, alanine, and small amounts of serine (Figure 1). A large proportion of the amino acids was found to contain heavy ${ }^{13} \mathrm{C}$ atoms, implying that both carbons from the diamond projectiles and ${ }^{12} \mathrm{C}$ target base plate participated in the plasma torch synthesis process. Mass-spectra also showed formation of much heavier organic products, among them, possibly, peptides composed of the above amino acids.

The most important results were generated by the numerous repeated chiral gas chromatographymass-spectrometry experiments. To perform them, an aqueous soluble fraction of organic products was subjected to acidic hydrolysis and the amino acids were converted into volatile isopropyl esters of $\mathrm{N}$-trifluoroacetyl derivatives. The chiral stationary phase in the $50 \mathrm{~m} \times 0.25 \mathrm{~mm}$ capillary column was Chirosil-Val from Altech. Glycine and alanine, enriched in ${ }^{13} \mathrm{C}$ to an extent of $40 \%$, showed up very clearly in the chromatogram. Mass spectra were recorded in both the single ion monitoring (SIM) and total ion current modes (TIC). The chiral imbalance of alanine was presented as the ratio of its L-and D-enantiomers. The fundamental result of the whole study was summed up by the authors as follows: “This measurement enabled us to calculate $\mathrm{L} / \mathrm{D}$ values for alanine based on peak integrals and peak amplitudes in both the SIM and total ion modes. Using SIM data, the values for alanine are L/D = 1.15 based on the peak amplitudes and $\mathrm{L} / \mathrm{D}=1.68$ from evaluating the integrals of peaks. For the total ion mode, the values for alanine are $\mathrm{L} / \mathrm{D}=2.5$ for the peak amplitudes and $\mathrm{L} / \mathrm{D}=2.4$ for integrals of 
peaks. Given the unavoidable background noise in the total ion mode, the $\mathrm{L} / \mathrm{D}$ values obtained for the SIM mode should be considered more reliable. Thus, the violation of symmetry could be in the range from 1.15 to 1.68" (Figure 2). This range corresponds to enantiomeric excess values between 7 and 25\%, which is similar to ee values found in meteorites and outperforms by far the values expected for all deracemization effects under action of cosmic polarized irradiations. Remarkably, alanine synthesized in the plasma torch reactor is noticeably enriched in the L-enantiomer, exactly as predicted from the hypothesis on homochirality of all elementary particles in Universe.

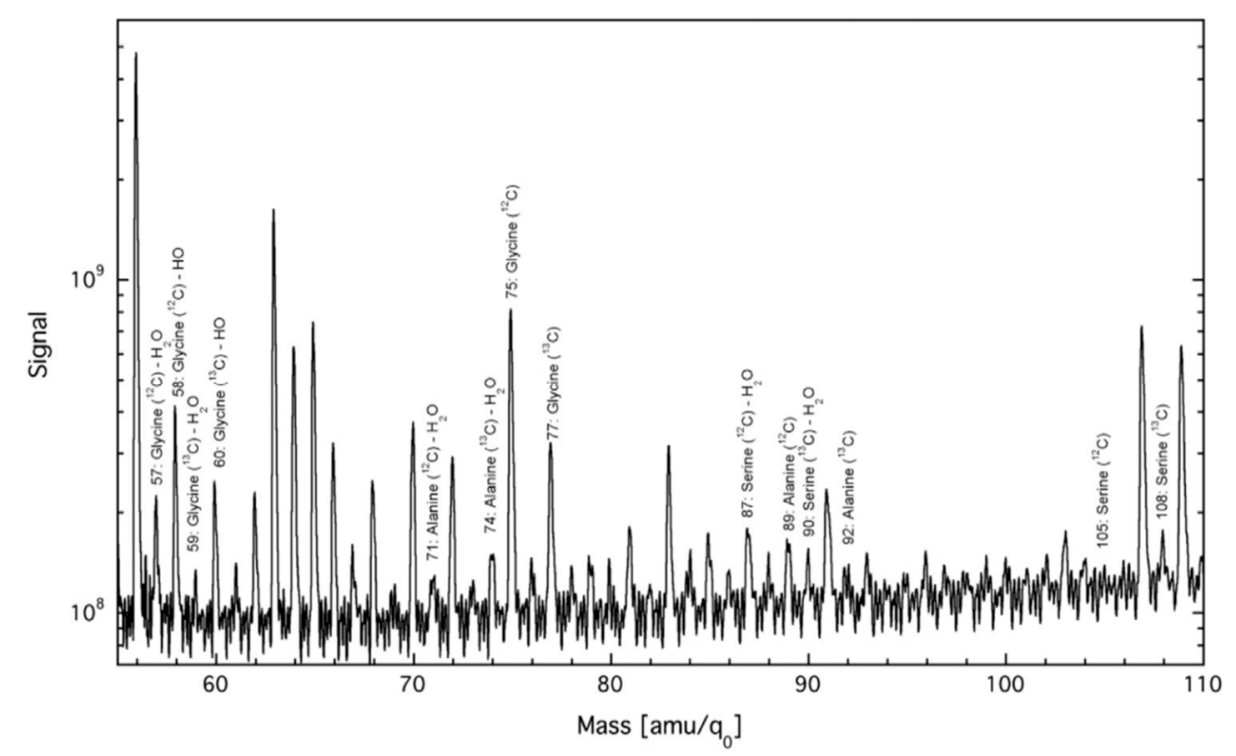

Figure 1. The amino acid-corresponding section of the MALDI mass spectra of impact products. The high content of ${ }^{13} \mathrm{C}$ isotope is evident. Notably, glycine peaks dominate over those of alanine (by 4.75 times) and serine, which is not characteristic of bioproteins. Source: Reference [51].

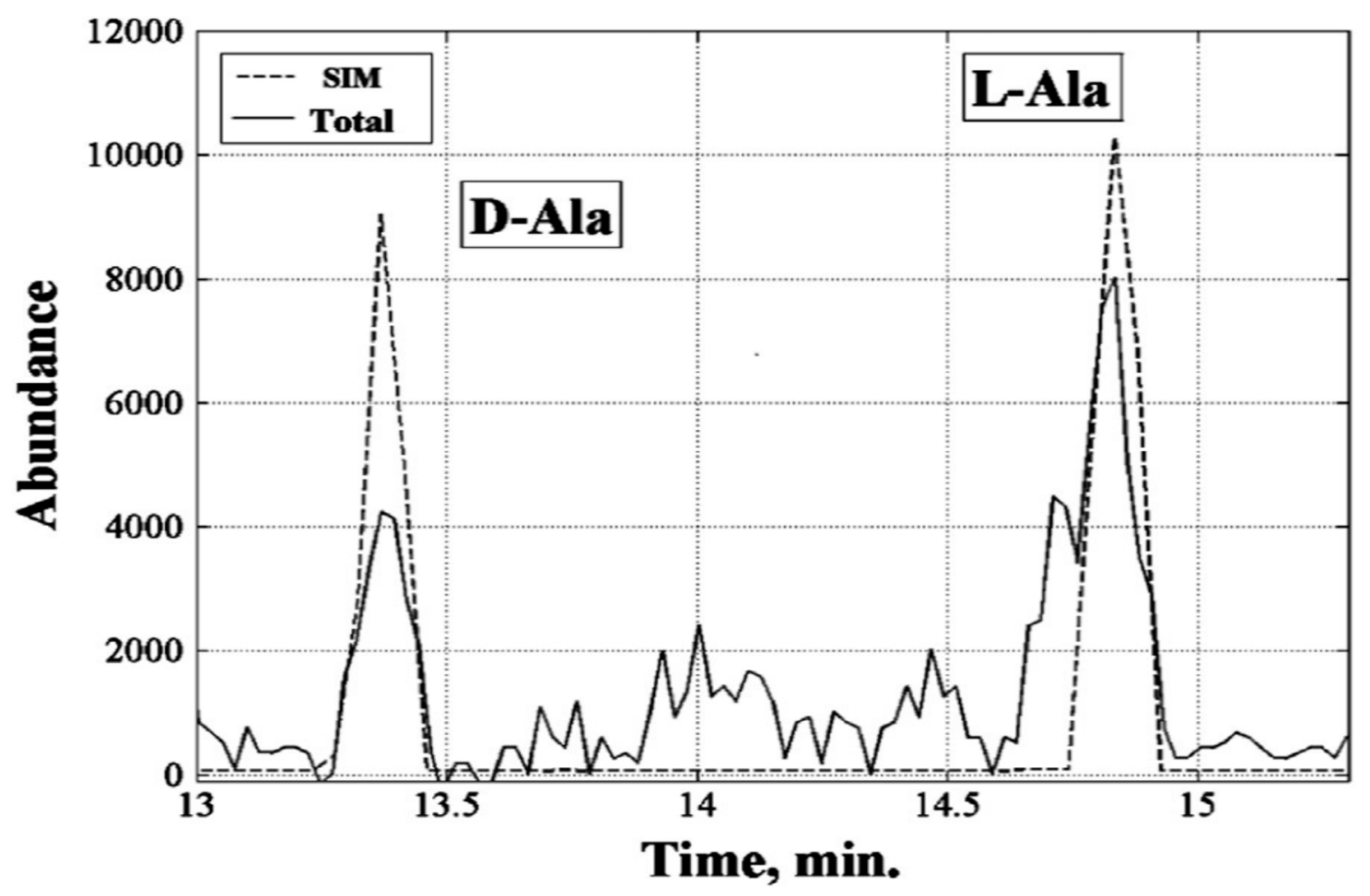

Figure 2. Section of the total chiral GC-MS profile with D-Ala and L-Ala peaks (retention times 13.3 and $14.7 \mathrm{~min}$, respectively) shown in SIM (140 amu) and TIC modes. Source: Reference [51]. 


\section{Conclusions}

Based on the above reliable and extremely informative experimental findings, one can speculate that many other $\alpha$-amino acids, including "non-natural" species, will be synthesized everywhere in the universe under conditions of much stronger hypervelocity collisions of cosmic bodies and that all of them will be characterized by the predominance of the $\mathrm{L}$ configuration. In a similar direct synthetic way or during subsequent chemical transformations of organic matter, D-isomers could become the predominating isomers of sugars. Mechanisms of further enantiomeric enrichment of some particular compounds [53] through the Soai-type asymmetric autocatalysis [15] and/or Viedma-type deracemization [4] are being intensively investigated but none of them could function efficiently and unidirectionally in a complex concentrated aqueous broth. Equally unknown remain the rules of selection of amino acids and sugars for creating first primitive self-reproducing organic systems. If one also takes into consideration the essential unique complex of physical conditions on the planet (such as the presence of water in its liquid form, a suitable atmosphere, sufficient magnetic field of the planet, and appropriate rotation rate of the latter around its axis and the central star, etc.) the fact of the emergence of life on the Earth appears a big mystery. Important is only that the general homochiral shift of organic building blocks and, hence, unique stereochemistry of living matter in the whole universe is predetermined by the parity violation of elementary particles, whereas realization of life forms on different planets may remain a matter of a single happy chance.

Funding: There is no extra funding.

Conflicts of Interest: The author declares no conflict of interest.

\section{References}

1. Eigenbrode, J.L.; Summons, R.E.; Steele, A.; Freissinet, C.; Millan, M.; Navarro-González, R.; Sutter, B.; McAdam, A.C.; Franz, H.B.; Glavin, D.P.; et al. Organic matter preserved in 3-billion-year-old mudstones at Gale crater, Mars. Science 2018, 360, 1096-1101. [CrossRef]

2. Goldanskii, V.I.; Kuz'min, V.V. Spontaneous mirror symmetry breaking in nature and the origin of life. Z. Phys. Chem. 1988, 269, 216-274. [CrossRef]

3. Chandrasekhar, S. Molecular homochirality and the parity-violating energy difference. A critique with new proposals. Chirality 2008, 20, 84-95. [CrossRef]

4. Viedma, C.; McBride, J.M.; Kahr, B.; Cintas, P. Enantiomer-specific oriented attachment: Formation of macroscopic homochiral crystal aggregates from a racemic system. Angew. Chem. 2013, 52, 10545-10548. [CrossRef]

5. Poinot, P.; Geffroy-Rodier, C. Searching for organic compounds in the Universe. Trends Anal. Chem. 2015, 65, 1-12. [CrossRef]

6. Myrgorodska, I.; Meinert, C.; Martins, Z.; Le Sergeant, L.; d'Hendecourt; Meierhenrich, U.J. Molecular chirality in meteorites and interstellar ices, and the chirality experiment on board the ESA Cometary Rosetta Mission. Angew. Chem. Int. Ed. 2015, 54, 1402-1412. [CrossRef]

7. Meinert, C.; Filippi, J.-J.; de Marcellus, P.; Le Sergeant, L.; d'Hendecourt; Meierhenrich, U.J. Why interstellar ices can be considered as precursors for prebiotic chemistry. ChemPlusChem 2012, 77, 186-191. [CrossRef]

8. Nuevo, M.; Milam, S.N.; Sandford, S.A. Molecules in organic residues produced from the ultraviolet photo-irradiation of pyrimidine in $\mathrm{NH}_{3}$ and $\mathrm{H}_{2} \mathrm{O}+\mathrm{NH}_{3}$ ices. Astrobiology 2012, 12, 295-314. [CrossRef]

9. Furukawa, Y.; Sekine, T.; Kobayashi, T.; Kakegawa, T. Nucleobase and amino acid formation through impacts of meteorites on the early ocean. Earth Planet. Sci. Lett. 2015, 429, 216-222. [CrossRef]

10. Martins, Z.; Price, M.C.; Goldman, N.; Sephton, M.A.; Burchell, M.J. Shock synthesis of amino acids from impacting cometary and icy planet surface analogues. Nat. Geosci. 2013, 6, 1045-1049. [CrossRef]

11. Esmaili, S.; Bass, A.D.; Cloutier, P.; Sanche, L.; Huels, M.A. Glycine formation in $\mathrm{CO}_{2}: \mathrm{CH}_{4}: \mathrm{NH}_{3}$ ices induced by $0-70 \mathrm{eV}$ electrons. J. Chem. Phys. 2018, 148, 164702. [CrossRef]

12. Pizzarello, S.; Schrader, D.L.; Monroe, A.A.; Lauretta, D.S. Large enantiomeric excesses in primitive meteorites and the diverse effects of water in cosmochemical evolution. Proc. Natl. Acad. Sci. USA 2012, 109, 11949-11954. [CrossRef] 
13. Glavin, D.P.; Dworkin, J.P. Enrichment of the amino acid L-isovaline by aqueous alteration on CI and CM meteorite parent bodies. Proc. Natl. Acad. Sci. USA 2009, 106, 5487-5492. [CrossRef]

14. Pizzarello, S.; Wang, Y.; Chaban, G.M. A comparative study of the hydroxy acids from the Murchison GRA 95229 and LAP 02342 meteorites. Geochim. Cosmochim. Acta 2010, 74, 6206-6217. [CrossRef]

15. Soai, K.; Kawasaki, T.; Matsumoto, A. The origins of homochirality examined by using asymmetric autocatalysis. Chem. Rec. 2014, 14, 70-83. [CrossRef]

16. Gabuzda, D. Astrophysics: The MAD world of black holes. Nature 2014, 510, 42-43. [CrossRef]

17. Bailey, J.; Chrysostomou, A.; Hough, J.H.; Gledhill, T.M.; McCall, A.; Clark, S.; Menard, F.; Tamura, M. Circular polarization in star-formation regions: Implications for biomolecular homochirality. Science 1988, 281, 672-674. [CrossRef]

18. Bailey, J. Astronomical sources of circularly polarized light and the origin of homochirality. Orig. Life Evol. Biosph. 2001, 31, 167-183. [CrossRef]

19. Meinert, C.; Filippi, J.J.; Nahon, L.; Hoffmann, S.V.; d’Hendecourt, L.; de Marcellus, P.; Bredehöft, J.H.; Thiemann, W.H.P.; Meierhendrich, U.J. Photochirogenesis: Photochemical models on the origin of biomelecular homochirality. Symmetry 2010, 2, 1055-1080. [CrossRef]

20. Meinert, C.; de Marcellus, P.; d’Hendecourt, L.; Nahon, L.; Jones, N.C.; Hoffmann, S.V.; Bredehöft, J.H.; Thiemann, W.H.P.; Meierhendrich, U.J. Photochirogenesis: Photochemical models on the absolute asymmetric formation of amino acids in interstellar space. Phys. Life Rev. 2011, 8, 307-330. [CrossRef]

21. Bartmess, J.E.; Pagni, R.M. A photochemical mechanism for homochirogenesis. Part 2. Chirality 2013, 25, 16-21. [CrossRef]

22. De Marcellus, P.; Meinert, C.; Nuevo, M.; Filippi, J.-J.; Danger, G.; Deboffle, D.; Nahon, L.; d'Hendecourt, L.L.S.; Meierhenrich, U.J. Non-racemic amino acid production by ultraviolet irradiation of achiral interstellar ice analogs with circularly polarized light. Astrophys. J. 2011, 727, L1-L6. [CrossRef]

23. Modica, P.; Meinert, C.; De Marcellus, P.; Nahon, L.; Meierhenrich, U.J.; D'Hendecourt, L.L.S. Enantiomeric excesses induced in amino acids by ultraviolet circularly polarized light irradiation of extraterrestrial ice analogs: A possible source of asymmetry for prebiotic chemistry. Astrophys. J. 2014, 788, 79-90. [CrossRef]

24. Cline, D.B. Supernova antineutrino interactions cause chiral symmetry breaking and possible homochiral biomaterials for life. Chirality 2005, 17, S234-S239. [CrossRef]

25. Boyd, R.N.; Kajino, T.; Onaka, T. Supernovae and the chirality of the amino acids. Astrobiology 2010, 10, 561-568. [CrossRef]

26. Boyd, R.N.; Kajino, T.; Onaka, T. Supernovae, neutrinos, and the chirality of the amino acids. Int. J. Mol. Sci. 2011, 12, 3432-3444. [CrossRef]

27. Famiano, M.; Boyd, R.; Kajino, T.; Onaka, T.; Koehler, K.; Hulbert, S. Determining Amino Acid Chirality in the Supernova Neutrino Processing Model. Symmetry 2014, 6, 909-925. [CrossRef]

28. Takahashi, J.-I.; Shinojima, H.; Seyama, M.; Ueno, Y.; Kaneko, T.; Kobayashi, K.; Mita, H.; Adachi, M.; Hosaka, M.; Katoh, M. Chirality emergence in thin solid films of amino acids by polarized light from synchrotron radiation and free electron laser. Int. J. Mol. Sci. 2009, 10, 3044-3064. [CrossRef]

29. Bonner, W.A.; van Dort, M.A.; Yearian, M.R.; Zeman, H.D.; Li, G.C. Polarized Electrons and the Origin of Optical Activity. Isr. J. Chem. 1976, 15, 89-95. [CrossRef]

30. Darge, W.; Laczko, I.; Thiemann, W. Stereoselectivity of beta irradiation of D,L-tryptophan in aqueous solution. Nature 1976, 261, 522-524. [CrossRef]

31. Akaboshi, M.; Noda, M.; Kawai, K.; Maki, H.; Kawamoto, K. Asymmetrical radical formation of D- and L0alanins irradiated with tritium-rays. Orig. Life 1982, 12, 395-399. [CrossRef]

32. Klabunowskii, E.I.; Pavlov, V.A. Homochiraity origine in nature: Possible versions. Curr. Org. Chem. 2014, 18, 93-114.

33. Naaman, R. Chirality—Beyond the Structural Effects. Isr. J. Chem. 2016, 56, 1010-1015. [CrossRef]

34. Fukue, T.; Tamura, M.; Kandori, R.; Kusakabe, N.; Hough, J.H.; Bailey, J.; Whittet, D.C.B.; Lucas, P.W.; Nakajima, Y.; Hashimoto, J. Extended high circular polarization in the Orion massive star forming region: Implications for the origin of homochirality in the Solar system. Orig. Life Evol. Biosph. 2010, 40, 335-346. [CrossRef]

35. Tachibana, S.; Huss, G.R.; Kita, N.T.; Shimoda, G.; Morishita, Y. ${ }^{60}$ Fe in Chondrites: debris from a nearby supernova in the early solar system? Astrophys. J. 2006, 639, L87-L90. [CrossRef] 
36. Cerf, C.; Jorissen, A. Is amino-acid homochirality due to asymmetric photolysis in space? Space Sci. Rev. 2000, 92, 603-612. [CrossRef]

37. Myer, Y.P.; MacDonald, L.H. Circular dichroism of L-tryptophan by an improved dichrograph. J. Am. Chem. Soc. 1967, 89, 7142-7144. [CrossRef]

38. Lee, T.D.; Yang, C.N. Question of parity conservation in the weak interaction. Phys. Rev. 1956, 104, $254-258$. [CrossRef]

39. Wu, C.S.; Ambler, A.; Hayward, R.W.; Hoppes, D.D.; Hudson, R.P. Experimental test of parity conservation in beta decay. Phys. Rev. 1957, 105, 1413-1415. [CrossRef]

40. Berger, R. Theoretical and Computational Chemistry; Peter, S., Ed.; Elsevier: Amsterdam, The Netherlands, 2004; Volume 14, pp. 188-288.

41. Davankov, V. Chirality as an inherent general property of matter. Chirality 2006, 18, 459-461. [CrossRef]

42. Emmons, T.P.; Reeves, J.M.; Forson, E.N. Parity-Nonconserving Optical Rotation in Atomic Lead. Phys. Rev. Lett. 1983, 51, 2089-2092. [CrossRef]

43. Brown, G.E.; Rho, M. On the manifestation of chiral symmetry in nuclei and dense nuclear matter. Phys. Rep. 2002, 363, 85-171. [CrossRef]

44. Avalos, M.; Babiano, R.; Cintas, P.; Jiménez, J.L.; Palacios, J.C. What does elementary chirality have to do with neutrinos? ChemPhysChem. 2002, 3, 1001-1003. [CrossRef]

45. Peskin, M. The matter with antimatter. Nature 2002, 419, 24-27. [CrossRef]

46. Zheng, X. Precision measurement of the weak charge of the proton. Nature 2018, 557, 171-172. [CrossRef]

47. Davankov, V.A. Inherent homochirality of primary particles and meteorite impacts as possible source of prebiotic molecular chirality. Russ. J. Phys. Chem. A 2009, 83, 1247-1256. [CrossRef]

48. Managadze, G. A new universal mechanism of organic compounds synthesis during prebiotic evolution. Planet. Space Sci. 2006, 55, 134-140. [CrossRef]

49. Davankov, V.A. Homochirality of organic matter-Objective law or curious incident? Israel J. Chem. 2016, 56, 1036-1041. [CrossRef]

50. Managadze, G. Plasma and collision processes of hypervelocity meteorite impact in the prehistory of life. Int. J. Astrobiol. 2010, 9, 157-174. [CrossRef]

51. Managadze, G.G.; Engel, M.H.; Getty, S.; Wurz, P.; Brinckerhoff, W.B.; Shokolov, A.G.; Sholin, G.V.; Terent'ev, S.A.; Chumikov, A.E.; Skalkin, A.S.; et al. Excessof L-alanine in amino acids synthesized in a plasma torch generated by a hypervelocity meteorite impact reproduced in the laboratory. Planet. Space Sci. 2016, 131, 70-78. [CrossRef]

52. Skalkin, A.S.; Suntsov, G.N.; Shokolov, A.G.; Yakhlakov, Y.V. Investigation of the crater-formation process in the case of a hypervelocity impact of an aluminium particle onto a massive barrier made of AMg-6 alloy. Kosmonavtika i Raketostroenie 2011, 62.

53. Ribó, M.; Blanco, C.; Crusats, J.; El-Hachemi, Z.; Hochberg, D.; Moyano, A. Absolute asymmetric synthesis in enantioselective autocatalytic reaction networks: Theoretical games, speculations on chemical evolution and perhaps a synthetic option. Chem. Eur. J. 2014, 20, 17250-17271. [CrossRef]

(C) 2018 by the author. Licensee MDPI, Basel, Switzerland. This article is an open access article distributed under the terms and conditions of the Creative Commons Attribution (CC BY) license (http:/ / creativecommons.org/licenses/by/4.0/). 